\title{
CONSIDERACIONES PRÁCTICAS EN LA ADQUISICIÓN PARCIAL DE ACCIONES DEL FUNDADOR DE UNA START-UP
}

\author{
RICARDO DE LA PIEDRA* \\ Universidad de Lima, Lima, Perú \\ BeATRIZ MELO** \\ Universidad Católica Andrés Bello, Caracas, Venezuela \\ Recibido: 28/12/2020 Aceptado: 11/1/2021 \\ doi: https://doi.org/10.26439/iusetpraxis2021.n053.5056
}

\begin{abstract}
RESUMEN. El auge de las start-ups a nivel internacional ha generado que la actividad transaccional respecto a tales emprendimientos sea cada vez más constante. El Perú no es ajeno a este fenómeno y, en muchas oportunidades, los paquetes accionarios materia de ventas implican que el accionariado de la start-up quede dividido entre fundadores e inversionistas, y es sumamente importante conocer qué términos principales deben pactarse tanto en el contrato de inversión como en el convenio de accionistas que se genere como consecuencia del ingreso del inversionista en la sociedad.
\end{abstract}

PALABRAS CLAVE: M\&A / start-ups / adquisición parcial / convenios de accionistas / compraventa de acciones

\section{PRACTICAL CONSIDERATIONS IN THE PARTIAL ACQUISITION OF SHARES OF THE FOUNDER OF A START-UP}

ABSTRACT. The boom of start-ups at an international level has generated that the transactional activity regarding these enterprises is each time more constant. Peru isn't a stranger to said phenomenon and, in many opportunities, the stock packages of these sales imply that the shares of the start-up are divided between founder and investor; it is extremely important to know what terms should be agreed on in the investment contract, as well as the shareholders agreement that would be generated as a consequence of the entry of investors in the stock company.

KEYWORDS: M\&A / start-ups / partial acquisitions / shareholders' agreements / SPA

\footnotetext{
* Abogado por la Universidad de Lima, Perú. MBA por IE Business School, España. Socio del área de M\&A del Estudio Muñiz.

** Abogada por la Universidad Católica Andrés Bello, Venezuela. LLM por la Universidad de Nueva York, Estados Unidos. Asociada del área de M\&A del Estudio Muñiz.
} 


\section{INTRODUCCIÓN}

Por su propia naturaleza, la mayoría de start-ups requieren en determinado momento de la participación de inversionistas que puedan aportar el capital adicional necesario para desarrollar las ideas o los productos y servicios que hayan dado origen a la empresa. En este sentido, los fundadores de una start-up saldrán al mercado en búsqueda de inversionistas que estén dispuestos a asumir el riesgo de invertir en un negocio nuevo o disruptivo a cambio de una participación en la sociedad, cuya expectativa será que el rendimiento sea acorde al riesgo de su inversión (evidentemente, no todas las empresas tienen riesgos iguales, así como no todos los emprendedores tienen perfiles iguales).

Un ejemplo emblemático de esta estructura es la inversión de 500000 dólares realizada en el 2004 por Peter Thiel en Facebook. La inversión inicial se hizo a cambio de una participación del 10,2 \% en la empresa, lo cual generó una ganancia para Thiel de más de mil millones de dólares tras la venta de la mayoría de sus acciones en el 2012. Sin embargo, es importante recordar que la inversión de Thiel fue todavía más fructífera para el fundador de Facebook, Mark Zuckerberg, cuyas acciones en la compañía actualmente están valorizadas en aproximadamente 68000 millones de dólares. No hay que ser abogado ni banquero de inversión para notar que los inversionistas son determinantes para el éxito de una start-up y el consecuente éxito de sus fundadores.

En línea con lo anterior, es importante revisar algunas de las principales consideraciones que debe tomar en cuenta el fundador de una start-up al momento de llegar a un acuerdo definitivo con un inversionista respecto a la venta de un porcentaje de su participación, así como el tratamiento que recibirá dicha inversión. Estas consideraciones tienen, al menos, dos aristas fundamentales: (i) los términos del contrato de inversión, y (ii) los términos del gobierno corporativo que regularán la relación como socios entre el fundador y el inversionista, recogidos en el convenio de accionistas.

\section{TÉRMINOS DEL CONTRATO DE INVERSIÓN}

Hay dos aspectos fundamentales que debe revisar cuidadosamente el fundador de una start-up al momento de acordar los términos de la adquisición de acciones de su empresa por un inversionista: (i) la valorización de las acciones y (ii) el régimen de indemnización del inversionista.

\section{Valorización de las acciones de la start-up}

El valor que se asigna a las acciones de la compañía determinará cuánto equity recibirá el inversionista a cambio de su inversión (mientras mayor valor se les asigne a las acciones, menor porcentaje recibirá el inversionista a cambio de su inversión). Esto es vital para el fundador a fin de garantizar que reciba la mayor inversión posible por el 
valor de la start-up, retenga la mayor cantidad de equity posible y mantenga el control para efectos de gobierno corporativo (como analizaremos más adelante).

En tal sentido, a pesar de que la valorización es un tema que se escapa de lo legal, por tratarse de un aspecto central de las inversiones en las start-ups tocaremos algunos puntos esenciales que se toman en cuenta y su eventual regulación en el contrato de inversión.

\section{Métodos de valorización}

Existen distintas formas de valorizar a una compañía. Los dos métodos principales son el método de comparables y el método de flujos descontados (discounted cash flow method, DCF).

\section{(i) Método de comparables}

En términos generales, el método de comparables utiliza un múltiplo del EBITDA (earnings before interest, taxes, depreciation and amortization) de una compañía para asignarle un valor. El múltiplo es determinado por la industria en la cual opera la compañía. El concepto es que las empresas del mismo sector deben valorizarse de la misma manera. La ventaja de este método es que otorga cierta certeza sobre la valorización de la empresa, ya que el EBITDA es un número objetivo calculado sobre la base de los estados financieros de la compañía (lo cual incluirá la negociación de los elementos de los estados financieros que se excluirán o agregarán a dicho cálculo al no corresponder al giro ordinario del negocio o tratarse de gastos extraordinarios). El EBITDA obtenido es multiplicado por un múltiplo fijado por el estudio de empresas comparables del mercado y el resultado es el valor empresa.

Sin embargo, este método tiene una desventaja importante: no toma en consideración el potencial de crecimiento de la compañía, sino su estado al momento de la adquisición (o el EBITDA histórico por determinados años, dependiendo de la negociación, lo que en un caso de emprendimiento generaría más dudas que certezas para un fundador). Indudablemente, el principal valor de una start-up proviene de su potencial, no de su estado al momento en el cual el inversionista decide invertir. Adicionalmente, resulta difícil fijar un múltiplo de EBITDA para una start-up particularmente novedosa, porque no existen comparables en el mercado; por ejemplo, ¿cuál empresa era comparable con Microsoft en el momento de su fundación en 1975? Por lo tanto, este método puede ser difícil de aplicar y puede presentar ciertas desventajas para el fundador.

\section{(ii) Método de DCF}

El método de DCF (flujo de caja descontado) consiste en valorizar la compañía con base en el valor actual de las ganancias que razonablemente se espera que pueda generar la 
compañía en el futuro. Así, se calculan los flujos de caja de la compañía hacia el futuro, descontando la tasa de retorno esperada para calcular su valor presente neto. Los elementos clave del modelo de valorización bajo comentario son las proyecciones financieras, la definición clara del concepto de flujo de caja y el costo del capital que se utilice para calcular una tasa apropiada de descuento (tradicionalmente se aplica un descuento mayor a las ganancias para contrapesar el alto riesgo vinculado al potencial fracaso del negocio). Los pasos que usualmente sigue el equipo financiero para este tipo de valorización son los siguientes:

- El primer paso es desarrollar la proyección de los estados financieros de la compañía. Como se puede intuir, esto no es tarea fácil, ya que implica tener o adquirir un conocimiento cercano de los resultados de la compañía, sus operaciones, riesgos asociados y una serie de asunciones relacionadas no solo con el objetivo, sino con la industria en la que esta desarrolla sus actividades. Esto es aún más complicado en industrias con alto potencial de innovación y disrupción.

- Una vez realizada la proyección, se debe determinar los flujos futuros de la compañía, esto es, los flujos "libres" que permanecen en la sociedad luego de deducir todos los gastos y costos relacionados con su actividad. Para mayor claridad, estos se deben entender como los recursos y flujos que no son necesarios para las operaciones y que, por lo tanto, están listos para ser distribuidos a los accionistas sin afectar el desarrollo del negocio de la empresa objetivo en el futuro.

- Determinados los montos relacionados con los flujos de caja, se les asigna un valor a futuro con base en el horizonte de la proyección (en el caso de emprendimientos, usualmente es de cinco años).

- Obtenido el valor de los flujos a futuro, se debe aplicar una tasa de descuento para traer dicho valor a la actualidad y obtener el valor presente de la compañía. La lógica es bastante simple: los flujos ocurrirán en el futuro y el valor se ha estimado a futuro, pero como la participación se está adquiriendo en el presente, se deben aplicar los principios del valor del dinero en el tiempo para traer al valor actual la estimación.

- Una vez aplicada la tasa de descuento, se obtendrá el valor actual de la compañía, sobre el cual comenzarán las negociaciones de adquisición.

Lo importante de este método es que le otorga valor a la compañía tomando en consideración su potencial para generar ganancias, lo cual es la esencia del valor de toda start-up. Sin embargo, la posibilidad de aplicar este método dependerá de la etapa en la que se encuentre la start-up, ya que es sumamente difícil predecir las ganancias que generará una compañía nueva para sus accionistas. 
Earn-outs

A diferencia de los métodos descritos en el punto anterior, el earn-out no es un método de valorización, sino uno para corregir o ajustar el valor de las acciones adquiridas por el inversionista con base en los resultados de la compañía en el tiempo después de la inversión.

En términos generales, esto consiste en un acuerdo según el cual, si la empresa alcanza determinados hitos de ganancias/crecimiento establecidos por las partes dentro de un tiempo determinado después de la venta/inversión, el inversionista deberá pagarle al vendedor un monto adicional calculado sobre la base de una fórmula. La ventaja de las cláusulas de earn-out es que permiten que el vendedor reciba un valor adicional sobre la base del éxito comprobado de la compañía.

Ahora bien, en el contexto especial de una start-up, es importante tomar en consideración que, en la mayoría de los casos, el dinero aportado por el inversionista a cambio de las acciones no se paga directamente al fundador, sino que se invierte en la compañía. Por lo tanto, dependiendo del estado de desarrollo de la start-up, el monto adicional calculado con base en la cláusula de earn-out puede ser invertido directamente en la compañía (si es preferible impulsar el crecimiento de la compañía) o puede ser pagado directamente al fundador.

\section{Conclusiones sobre valorización}

El propósito de este trabajo no es determinar el mejor método de valorización de una startup, sino describir los métodos principales de valorización y ajuste a fin de que un fundador conozca las metodologías que podrán aplicar los asesores financieros de las partes.

En este contexto, es importante recordar que la valorización de una start-up que sea exitosa, muy probablemente, no tomará en cuenta todo el potencial de la start-up (de lo contrario, Peter Thiel hubiese pagado mucho más de 500000 dólares por el equity de Facebook). El equilibrio del acuerdo entre las partes se encuentra en la necesidad del fundador de conseguir inversionistas para desarrollar una idea novedosa y el gran riesgo que asume el inversionista al invertir en dicha idea.

\section{Indemnización del inversionista}

Cuando un comprador adquiere acciones de una compañía, tradicionalmente solicita que el vendedor realice una serie de declaraciones y garantías sobre el estado de la compañía en el contrato ("Declaraciones"). En caso de que alguna de estas declaraciones no sea cierta por hechos previos a la fecha de adquisición, el vendedor tendrá que indemnizar al comprador (dentro de ciertas limitaciones). Este régimen también es aplicable a la entrada de un inversionista a una start-up. El inversionista estará dispuesto a asumir 
los riesgos vinculados a su inversión, pero no las contingencias que puedan surgir por hechos ocurridos antes de que se convirtiera en accionista de la empresa.

En adición a ello, es usual que un inversionista solicite el cumplimiento de determinadas obligaciones del emprendedor (directas o de generar que la compañía cumpla algún hecho u obligación determinada). A manera de ejemplo, pensemos que un porcentaje $X$ del monto de la inversión sea destinado a R\&D, modernización, implementación de canales o lo que su imaginación dicte con base en la gama de start-ups que existen actualmente.

En línea con lo anterior, el contrato de inversión contendrá lo que se denomina el régimen de indemnización, con características muy similares al de un contrato de adquisición, en las que el inversionista responderá principalmente por (i) la falsedad o inexactitud de las declaraciones, (ii) el incumplimiento de obligaciones asumidas en el contrato, y (iii) hechos dolosos o fraudulentos.

\section{Declaraciones y determinación de responsabilidad}

El fundador tendrá que otorgar una serie de declaraciones en el contrato de inversión sobre el estado de la compañía al momento de la inversión y transferencia del equity. El contenido de las declaraciones dependerá del rubro en el que opera la start-up. Por ejemplo, en el caso de una start-up de tecnología, un inversionista requerirá que el fundador otorgue declaraciones robustas en materia de propiedad intelectual, a fin de que sea indemnizado en caso de que se materialice un daño con motivo de un reclamo sobre la propiedad de la tecnología desarrollada por la empresa.

Igualmente, un inversionista probablemente solicitará declaraciones generales que aplican a todas las empresas, tales como declaraciones sobre el cumplimiento de las normas aplicables en materia tributaria, así como las denominadas declaraciones fundamentales, que versan sobre hechos esenciales de la transacción (propiedad de las acciones, capacidad del fundador para contratar, entre otras).

Sobre la base de lo anterior, el fundador deberá indemnizar al inversionista en caso de que la compañía o el inversionista sufran un daño con motivo de la falsedad o inexactitud de alguna de las declaraciones sobre la compañía. Por ejemplo, si la declaración establece que "la compañía ha cumplido con todas sus obligaciones tributarias" y, con posterioridad al cierre del contrato de inversión, la administración tributaria obliga a la compañía al pago de un impuesto adeudado desde antes de la fecha de cierre, el fundador deberá indemnizar al inversionista (sujeto a las limitaciones generales detalladas en la siguiente sección). 
Limitaciones a la responsabilidad del fundador por declaraciones

Existen dos limitaciones principales a la responsabilidad del fundador por las contingencias de la compañía: (i) limitaciones de tiempo y (ii) limitaciones de monto. Las limitaciones de tiempo establecen un plazo máximo durante el cual el fundador es responsable ante el inversionista por los daños producidos por la falsedad o inexactitud de las declaraciones otorgadas. Tradicionalmente, el límite en el tiempo de la responsabilidad es negociado por las partes utilizando el plazo de prescripción legal como punto de partida, el cual el fundador intentará reducir.

La segunda limitación principal es la del monto de responsabilidad. El contrato de inversión establecerá un monto máximo de responsabilidad (conocido como CAP) y, en muchas ocasiones, se establecerá un monto individual (conocido como basket) de responsabilidad y un monto mínimo de responsabilidad (conocido como de minimis o minibasket). Si se aplican todas estas limitaciones al mismo tiempo, los límites de responsabilidad funcionarán según el siguiente ejemplo con montos aleatorios: cualquier daño deberá ser de al menos 10000 dólares (minibasket) para poder ser reclamable y poder acumularse junto con otros daños equivalentes hasta llegar a 100000 dólares (basket). A partir del monto del basket, el fundador será responsable desde el primer dólar hasta el monto del daño (cuando el basket no es deducible) o desde montos que excedan 100000 dólares hasta el monto del daño (cuando el basket sí es deducible). En ningún caso (salvo supuestos de dolo, fraude o falsedad de declaraciones fundamentales), el fundador responderá por un monto superior al CAP.

\section{Capacidad de negociación de las partes}

Hemos descrito el régimen general de indemnización de un contrato de inversión que explica la responsabilidad que permanece con el socio fundador aún después del ingreso de un inversionista al start-up.

Sin embargo, es importante tomar en consideración que los términos definitivos que se incluirán en cualquier contrato de compraventa de acciones o contrato de inversión dependerán de la capacidad de negociación de las partes y de la situación de cada una de ellas. En este contexto, un fundador que necesita los fondos de un inversionista de manera urgente para potenciar una start-up de manejo desordenado, incipiente o con un perfil del fundador complicado puede tener una capacidad de negociación reducida frente a un inversionista sofisticado. Caso contrario es el de una start-up con un futuro promisorio, en orden y con potenciales socios competentes y enfocados en el desarrollo de la compañía y su modelo de negocios. 


\section{GOBIERNO CORPORATIVO POSINVERSIÓN}

Además de definir los términos del contrato de inversión, el socio fundador de una startup debe pensar también en la regulación de su relación con el inversionista como socios de la compañía y los términos del gobierno corporativo de la empresa. El gobierno corporativo se refiere al régimen mediante el cual se regula el control y operación de una empresa. Este régimen se establece en dos documentos fundamentales: los estatutos y el convenio de accionistas.

Asumiendo que los estatutos de la empresa se mantienen dentro de un modelo estándar, analizaremos los acuerdos usuales que negocian los accionistas en el convenio de accionistas, enfocándonos en el contexto de las start-ups y el ingreso de un inversionista a la empresa.

\section{Concepto de control}

Antes de examinar las distintas cláusulas que se pueden establecer en los convenios de accionistas, es importante entender el concepto de control dentro de una empresa.

Según la normativa peruana, existen tres órganos fundamentales en una sociedad anónima: la junta de accionistas, el directorio y la gerencia. Estos tres órganos ejercen distintas funciones dentro de la empresa y se les puede asignar distintos poderes para balancear la toma de decisiones y el manejo del día a día del negocio. Sin embargo, en toda circunstancia, la junta de accionistas será el órgano supremo y decisorio de la sociedad. Por lo tanto, los porcentajes de acciones que posee cada accionista son sumamente relevantes.

En el Perú, por los términos de la Ley 26887, Ley General de Sociedades, un accionista controla la empresa de forma absoluta si sostiene al menos el $60 \%$ del accionariado. Por este motivo, un accionista minoritario en una start-up (ya sea el inversionista o el fundador después de la adquisición) siempre solicitará la firma de un convenio de accionistas que le otorgue ciertos derechos de minoría que no le otorga la ley en términos de toma de decisiones y otros aspectos de la vida de la compañía. De esta manera, el accionista puede ejercer cierto control a través de "derechos de veto" o supermayorías.

Ahora bien, como explicaremos a continuación, el convenio de accionistas también puede ser beneficioso para el fundador, ya que se pueden pactar ciertos derechos a favor del socio mayoritario o que benefician al socio fundador que no están previstos en la Ley General de Sociedades. Esto, finalmente, dependerá del monto de la adquisición parcial de parte del inversionista y si esta le otorga o no una posición de control. 


\section{Mayorías calificadas y derechos de veto}

A favor de los intereses del accionista minoritario (para efectos del presente escenario, el inversionista), es posible establecer mayorías más altas que las calificadas para la toma de ciertas decisiones por la junta de accionistas y derechos de veto sobre ciertas materias.

Las mayorías calificadas se refieren al aumento de los porcentajes de quorum y votación necesarios para tomar una decisión dentro de la junta de accionistas. Como comentamos en el punto anterior, según la Ley General de Sociedades, un accionista puede tomar todo tipo de decisiones de forma unilateral si sostiene el $60 \%$ del accionariado. Sin embargo, es posible aumentar este porcentaje para ciertas materias a través de lo que se conoce coloquialmente como "supermayorías". Por ejemplo, si el fundador sostiene el $70 \%$ y el inversionista mantiene el $30 \%$ de la empresa, las partes podrán acordar que la toma de algunas decisiones requerirá el quorum y voto favorable del $71 \%$ del accionariado. De esta forma, mientras los porcentajes se mantengan en 70/30, el fundador no podrá tomar estas decisiones de forma unilateral, sino que requerirá siempre la presencia y voto favorable del inversionista.

Tradicionalmente, se solicitan supermayorías para ciertas decisiones particularmente importantes en la empresa, como la modificación de los estatutos, modificaciones del capital social, elección de gerencias, disposición de activos importantes, toma de financiamientos fuera de la política de endeudamiento, aprobación o modificación del plan de negocios, plan de negocios, política de financiamiento, entre otros. La materia dependerá de la sensibilidad de los accionistas, del porcentaje adquirido y, claramente, de la posición del emprendedor, la compañía y el inversionista al momento de negociar el convenio.

\section{Elección de gerencias clave y del directorio}

El gerente general, las gerencias clave y el directorio de la compañía ejercen funciones más operativas dentro de la empresa en comparación con la junta de accionistas. A fin de mantener cierto grado de control sobre las operaciones, el accionista minoritario o inversionista puede solicitar que se establezca un régimen según el cual (i) designe un número de directores y (ii) tenga la potestad de nominar o alternar la facultad de designar al gerente general o alguna gerencia clave.

Existen diversas fórmulas para establecer el régimen de nombramiento de directores. El directorio puede estar conformado por un número par de integrantes y cada accionista tendrá derecho a nombrar el mismo número de directores. Igualmente, si el accionista minoritario no exige tener tanto control sobre las operaciones de la compañía, sino ejercer un grado de influencia, puede establecerse que el directorio estará conformado por un número impar de directores y el accionista minoritario designará a la minoría de directores. 
En relación con el nombramiento del gerente general, es posible establecer que las partes se turnarán en esta designación o, en todo caso, que las partes acordarán previamente quién será nominado como gerente general de la compañía. Lo mismo puede ocurrir con gerencias clave, como la de administración y finanzas o de R\&D (dependiendo del rubro de la empresa).

La regulación de este punto varía mucho en cada caso, porque depende de las circunstancias específicas de cada inversión. En el caso de una start-up, el grado de control que quiera tener el inversionista dependerá del tipo de inversionista que sea y de lo que quiera aportar a la operación. Habrá inversionistas ángeles que únicamente quieran aportar capital y permitir que el fundador maneje las operaciones de la compañía, mientras que otros inversionistas, como los fondos de venture capital, querrán aportar cierto grado de know-how y contactos para asegurar el éxito y crecimiento de la empresa para el eventual exit.

\section{Drag along y tag along}

Dos cláusulas que tradicionalmente se incluyen en los convenios de accionistas son las de drag along y tag along. La de drag along es solicitada usualmente por el accionista mayoritario (el fundador, en nuestro caso) y la de tag along es requerida comúnmente por el accionista minoritario (el inversionista, en nuestro caso). Estas disposiciones son clave en un convenio de accionistas de una start-up porque están relacionadas con la exit strategy de los socios.

\section{Drag along}

La cláusula de drag along establece que, si un accionista desea vender sus acciones a un tercero, puede obligar al otro accionista a vender sus acciones al mismo tercero en los mismos términos y condiciones. Es decir, si el accionista A es titular del $65 \%$ de las acciones y recibe una oferta de un tercero para adquirir sus acciones, pero dicho tercero desea comprar el $100 \%$ (muchos compradores no están dispuestos a permanecer en la compañía con un accionista minoritario), el accionista A puede obligar al accionista B (titular del $35 \%$ restante) a venderle sus acciones al tercero interesado en las mismas condiciones; así se obtiene un mejor valor empresa y, por ende, un mayor precio por las acciones en venta.

Como se puede inferir, la cláusula de drag along es esencial para un socio fundador que permanecerá como accionista mayoritario, porque aumenta sus posibilidades de liquidar su inversión en la start-up una vez que haya incrementado considerablemente el valor de la empresa; es decir, fortalece su exit strategy. 
Tag along

La cláusula de tag along dispone que si un accionista desea vender sus acciones a un tercero, el otro accionista puede adherirse a la venta y hacerlo de manera conjunta. Por lo tanto, en el escenario descrito en el párrafo anterior, si el accionista A recibe una oferta de un tercero y quiere proceder con la venta únicamente de sus acciones, el accionista $B$ puede condicionar la venta a que el tercero también adquiera sus acciones en los mismos términos y condiciones. Es decir, el accionista mayoritario no podrá salir de la compañía y dejar al accionista minoritario con el adquiriente.

La cláusula de tag along también es sumamente relevante para la exit strategy de un inversionista, porque los escenarios de venta del $100 \%$ de una compañía son mucho más frecuentes que el escenario de venta de un porcentaje minoritario. Por lo tanto, podrá capitalizar su inversión con mayor facilidad ante una buena oferta de un tercero.

\section{Call option y put option}

Las cláusulas de call option y put option se incluyen en un gran número de convenios de accionistas. La call option otorga a un accionista el derecho a obligar a otro accionista a venderle sus acciones, mientras la put option le otorga a un accionista el derecho a obligar a otro accionista a comprarle sus acciones. Existen dos supuestos principales que pueden gatillar el ejercicio de cualquiera de estas cláusulas: (i) el tiempo y (ii) algún evento significativo en la empresa o la relación de los accionistas.

El primer supuesto es el tiempo. Los accionistas acuerdan que, dentro de un número determinado de años después de la firma del convenio, alguno de los accionistas tendrá una put option (normalmente este derecho lo tendrá el accionista minoritario) o una call option (normalmente este derecho lo tendrá el accionista mayoritario). La lógica detrás de este mecanismo es que el accionista minoritario podrá aprovecharse del crecimiento de la empresa después de un tiempo determinado y vender sus acciones restantes a un precio mayor a lo que las hubiese vendido en la fecha inicial. Por su parte, el accionista mayoritario tendrá una call option para adquirir el porcentaje restante de la empresa y tener el control absoluto de sus operaciones (liberándose de los términos del convenio de accionistas). En el contexto de una start-up, cualquier estructura de put/call option va a depender de la exit strategy de los accionistas.

El segundo supuesto que puede gatillar una put/call option es un evento significativo para la empresa o en la relación de los accionistas. Dos ejemplos emblemáticos son el cambio de control de algún accionista o el incumplimiento de algún accionista del término del convenio de accionistas. En cualquiera de estos supuestos, la relación entre los accionistas puede verse afectada, por lo cual un accionista puede querer adquirir las acciones del otro accionista (call option) o, posiblemente, salir de la compañía y venderle sus acciones al otro accionista (put option). 
En cualquier caso, es posible establecer ciertos términos para el ejercicio de la call option o de la put option, tales como un monto mínimo de adquisición, la valorización de las acciones por un auditor independiente, la ventana de ejercicio, entre otros.

\section{Lock-up}

La cláusula de lock-up es común en aquellos convenios de accionistas en los cuales se quiere mantener cierta estabilidad de socios por un tiempo determinado. El lock-up prohíbe que uno o varios de los accionistas vendan sus acciones por un cierto tiempo (en el Perú, el lock-up no puede tener una duración mayor de diez años, según la Ley General de Sociedades).

Esta cláusula puede ser útil para el fundador en la medida que quiera mantener cierta estabilidad mientras la empresa está en un proceso de crecimiento y asegurar que el inversionista no venderá sus acciones a un tercero que el fundador no quiera tener como socio. Igualmente, el lock-up puede ser útil para el inversionista porque garantiza que el socio fundador no saldrá de la empresa antes de tiempo y continuará desarrollando el concepto de la start-up durante un periodo determinado.

\section{Deadlock}

El mecanismo de deadlock rige aquellas circunstancias en las cuales, de acuerdo con los términos del estatuto o el convenio de accionistas, se requiere del voto de ambos accionistas para tomar una decisión en la junta de accionistas y los accionistas no logran llegar a un acuerdo.

En este caso, normalmente se establece un plazo en el que los accionistas pasarán por un proceso de mediación (no vinculante) a fin de llegar a un acuerdo sobre la materia objeto del conflicto. Si aun así los accionistas no pueden llegar a un acuerdo, normalmente se establece (i) que alguno de ellos tiene una call option (el accionista mayoritario), o (ii) que se llevará a cabo un mecanismo de shotgun. Este implica que un accionista presentará una propuesta con el monto en que estaría dispuesto a comprar las acciones del otro, para que este último decida si vende o si se las compra al accionista que dio la oferta a ese precio. Hay otros mecanismos adicionales que las partes pueden pactar para solucionar un deadlock, siendo lo importante que el desacuerdo sustancial no haga perder valor a la compañía y, por ende, a sus intereses en la misma.

En un deadlock, el accionista con mayor capacidad económica tendrá una ventaja frente al otro accionista. En caso de que el accionista con mayor capacidad económica sea el inversionista, es importante que el socio fundador asegure un precio mínimo por la compra de sus acciones (por ejemplo, EBITDA de la fecha de firma del convenio por un múltiplo) e intente limitar los casos en los cuales sea posible gatillar el mecanismo de deadlock en el convenio de accionistas. 


\section{Derecho de adquisición preferente}

Esta conocida cláusula establece que ninguno de los accionistas podrá venderle su participación a un tercero sin antes ofrecérsela al otro accionista en las mismas condiciones de venta. Únicamente en caso de que el otro accionista no desee ejercer su derecho de adquisición preferente, el accionista vendedor podrá proceder con la venta al tercero en los mismos términos en que las ofreció al otro accionista.

El propósito de esta cláusula es similar al del lock-up. Los accionistas quieren asegurar que se mantendrá cierta estabilidad dentro de la empresa y, sobre todo, que no estarán sujetos a participar como socios dentro de la compañía con terceros a los cuales no eligieron.

En el contexto de una start-up, esto puede ser particularmente importante para el socio fundador, ya que, en caso de que el inversionista desee vender sus acciones porque quiere invertir en otros proyectos o no considera que se ha cumplido con ciertos hitos de crecimiento, el socio fundador puede recuperar el $100 \%$ de la compañía y no estar sujeto a la entrada de un tercero que no ha elegido.

\section{Derecho de primera oferta}

El derecho de primera oferta establece que los accionistas deben ofrecerle sus acciones en venta al otro accionista antes de ofrecer sus acciones en venta a algún tercero. Se diferencia del derecho de adquisición preferente en que esta cláusula le otorga al titular el derecho de realizar la primera oferta sobre las acciones del otro accionista, antes de que exista un tercero interesado. En cambio, el derecho de adquisición preferente permite que el titular del derecho compre las acciones después de que un tercero interesado ha presentado una oferta.

El propósito de esta cláusula es similar al del derecho de adquisición preferente y el lock-up: mantener el accionariado en manos de los mismos accionistas o darles herramientas a los accionistas para evitar la entrada de terceros a la empresa.

\section{Política de dividendos}

Es muy común establecer una política de dividendos en el convenio de accionistas, la cual regulará el régimen de distribución de dividendos de la empresa (frecuencia y porcentajes).

Esta política está íntimamente vinculada al plan de negocios, que se tratará en el punto siguiente, ya que la distribución de dividendos dependerá del plan de negocios que se haya establecido para la start-up. En emprendimientos, es común que la política de dividendos implique la no repartición de dividendos por un plazo determinado, ya que tanto el fundador como el inversionista no están interesados en el retorno inmediato de 
su inversión, sino en el crecimiento a largo plazo del emprendimiento. Por lo tanto, la política de dividendos probablemente establecerá que por un periodo determinado no se distribuirán dividendos y las utilidades devengadas se invertirán en la empresa.

\section{Plan de negocios}

Los accionistas suelen preparar un plan de negocios para la compañía. En este plan se establece cuáles son los objetivos para la empresa y los planes comerciales que tienen los accionistas para el desarrollo de la compañía.

Esto es particularmente importante en el caso de una start-up porque el crecimiento de la empresa es el objetivo fundamental de los accionistas, y la existencia de un plan de negocios aclara la ruta que se seguirá y evita futuros conflictos de los accionistas en relación con el camino que se tomará para lograr dicho crecimiento. Cualquier modificación del plan de negocios usualmente es materia de una supermayoría o derecho de veto, ya que afectará directamente al accionista minoritario.

\section{Política de financiamiento}

La política de financiamiento está íntimamente vinculada al plan de negocios, y establece los medios que utilizará la compañía para financiar sus actividades. Para un accionista que tenga menor capacidad económica que otros accionistas, la política de financiamiento es clave porque puede establecer que la compañía financiará sus actividades mediante la obtención de deuda u otros medios distintos de aumentos de capital o aportes de los accionistas, evitando así la dilución.

Una situación difícil se presenta cuando la sociedad necesita capital para operar, pero uno de los accionistas no cuenta con los medios para hacer el aporte y, a la vez, ese acuerdo requiere el voto de este último, de acuerdo con el convenio. Usualmente este tipo de impases se regula como una situación de deadlock, pero inexorablemente el tiempo que puede transcurrir entre la solución de la situación accionaria de la empresa y la necesidad de la inyección de capital repercutirá de manera negativa en la sociedad. En ese sentido, es importante considerar las consecuencias de la política de financiamiento, así como tener una lectura clara de las expectativas económicas y el perfil tanto del emprendedor como del inversionista.

\section{Confidencialidad}

La cláusula de confidencialidad, que sujeta a los accionistas a no revelar información confidencial a terceros es particularmente importante en el caso de las start-ups porque estas se definen por ser empresas que han desarrollado un producto o servicio novedoso, cuyas especificaciones son altamente confidenciales. Por lo tanto, es esencial proteger la información confidencial o trade secrets mediante esta disposición. 
Cabe señalar que la obligación de confidencialidad, en muchas ocasiones, obliga a los accionistas aun después de que dejen de ser accionistas de la empresa. Por lo tanto, en caso de que el inversionista decida vender sus acciones, no podrá revelar información confidencial que haya adquirido sobre la empresa y sus operaciones, lo que potencialmente disminuiría el valor de la start-up.

\section{Preferencia de negocios}

La cláusula de preferencia de negocios es importante en el caso de una start-up porque el crecimiento de esta radica en el éxito de una idea novedosa que, durante esta etapa de crecimiento, no tiene competencia. La cláusula establece que si alguno de los accionistas es invitado o tiene la intención de participar en el desarrollo de algún negocio que compita con el negocio de la compañía, se encontrará obligado a invitar al otro accionista a participar conjuntamente en el nuevo negocio.

El accionista fundador podrá evitar que el inversionista invierta en otras start-ups que compitan con la compañía sin su participación e, igualmente, el inversionista podrá evitar que el accionista fundador inicie otros proyectos novedosos que compitan con el proyecto original sin la participación del inversionista.

Esta cláusula es una alternativa a la cláusula de no competencia, que evitaría que las partes inviertan en cualquier negocio que compita con la start-up de forma absolutamente restrictiva. La cláusula de no competencia es muy rígida en el contexto de una start-up porque muchas veces los inversionistas son inversionistas institucionales que se dedican a invertir en varios negocios, por lo cual no aceptan cláusulas de no competencia como política institucional. La cláusula de preferencia de negocios, en cambio, permite que las dos partes participen en un negocio en caso de que quieran participar, pero, si uno no está interesado, todavía permite que la otra parte invierta en el otro negocio.

\section{REFLEXIÓN FINAL}

La relación entre un socio fundador de una start-up y un inversionista nace con la inversión inicial y adquisición de acciones. Este momento inicial se rige principalmente por dos aspectos clave: (i) el valor que se le asignará a las acciones de la start-up y (ii) la estructura del régimen de indemnización del inversionista por contingencias previas de la start-up. Sin embargo, la regulación de la relación de las partes posinversión tiene igual o mayor importancia, ya que esta relación puede tener un gran impacto sobre el éxito de la start-up, el valor y la exit strategy de las partes. Siendo esto así, los términos del convenio de accionistas deberán negociarse tomando en consideración el papel que juega cada parte en la empresa y la protección que requiere por sus condiciones económicas y sus intenciones de permanencia. 
